\title{
A Simple Model for the Disintegration of Highly Charged Solvent Droplets during Electrospray Ionization
}

\author{
Lars Konermann \\ Department of Chemistry, The University of Western Ontario, London, Ontario, Canada
}

This work uses a minimalist model for deciphering the opposing effects of Coulomb repulsion and surface tension on the stability of electrosprayed droplets. Guided by previous observations, it is assumed that progeny droplets are ejected from the tip of liquid filaments that are formed as protrusions of an initially spherical parent. Nonspherical shapes are approximated as assemblies of multiple closely spaced beads. This strategy greatly facilitates the calculation of electrostatic and surface energies. For a droplet at the Rayleigh limit the model predicts that growth of a very thin filament is a spontaneous process with a negligible activation barrier. In contrast, significant barriers are encountered for the formation of larger diameter filaments. These different barrier heights favor highly asymmetric droplet fission because the dimensions of the filament determine those of the ejected droplet(s). Substantial charge accumulation occurs at the filament termini. This allows each progeny droplet to carry a significant fraction of charge, despite its very small volume. In the absence of a long connecting filament, relieving electrostatic stress through progeny droplet emission would be ineffective. The model predicts the prevalence of fission events leading to the formation of several progeny droplets, instead of just a single one. Ejection bursts are followed by collapse back to a spherical shape. The resulting charge depleted system is incapable of producing additional progeny droplets until solvent evaporation returns it to the Rayleigh limit. Despite the very simple nature of the model used here, all of these predictions agree with experimental data. (J Am Soc Mass Spectrom 2009, 20, 496-506) (C) 2009 Published by Elsevier Inc. on behalf of American Society for Mass Spectrometry

$\mathrm{E}$ lectrospray ionization (ESI) mass spectrometry (MS) has evolved into one of the most versatile and widely used analytical techniques. The ESI process itself has been subject of numerous studies (see, e.g., [1-12] and references therein). Analyte solution is passed through a metal capillary to which high voltage has been applied, and solvent droplets emanate from the tip of a Taylor cone at the capillary outlet [11]. Each droplet carries a net charge due to protons or other cationic species that reside at the air/liquid interface. Solvent evaporation and droplet fission are fundamental elements of the ESI process. Evaporation increases the surface charge density to a point where fission occurs. The disintegration process triggered in this way is highly asymmetric, leading to the formation of several small progeny droplets that carry away only a small fraction of mass, but a disproportionately high amount of charge [13, 14]. High-speed imaging is an important tool for gaining insights into the breakup mechanism [15, 16]. More recently, the rapid solidification of charged nanodroplets using sol-gel techniques has provided a means to capture transient droplet

Address reprint requests to Dr. L. Konermann, Department of Chemistry, The University of Western Ontario, London, Ontario N6A 5B7, Canada. E-mail: konerman@uwo.ca shapes and study them by microscopy [17]. Also, molecular dynamics simulations represent an interesting approach in this area $[9,10]$. All those studies reveal that disintegration proceeds through intermediate structures where a parent droplet carries one or two thin protrusions that can be considerably longer than the parent diameter. Offspring droplets are ejected from the tip of these liquid filaments in a manner that is reminiscent of processes occurring at the tip of a Taylor cone [6]. After these emission "bursts", the charge-depleted residual parent returns to an approximately spherical shape $[15,16,18]$. Repeated evaporation and breakup of the fission products ultimately result in the formation of nanometer-sized droplets from which gas-phase analyte ions are produced [1].

Progeny droplets are formed predominantly from the outermost layers of their parent, leading to an enrichment of species with high surface affinity $[13,19$, 20]. In contrast, compounds with low surface affinity accumulate in the charge-depleted residual parent, from where ionization is inefficient [21, 22]. Two limiting cases are commonly discussed for the final step by which ions are released into the gas phase from nanometer-sized progeny droplets. According to the charged residue model (CRM), solvent evaporation to dryness releases the analyte, which retains some of the droplet's charge
(C) 2009 Published by Elsevier Inc. on behalf of American Society for Mass Spectrometry. $1044-0305 / 09 / \$ 32.00$

doi:10.1016/j.jasms.2008.11.007
Published online November 21, 2008 Received October 7, 2008 Revised November 12, 2008 Accepted November 14, 2008 
[23]. An alternative framework, the ion evaporation model (IEM), stipulates that charged analytes can be ejected from the droplet surface [24, 25]. It has been suggested that large biomolecular ions are formed predominantly via the CRM mechanism [2, 26-29], and that the IEM is operative for relatively small analytes [1]. In addition, proposals for scenarios involving elements of both models have been put forward [30, 31].

A solvent droplet is said to be at the Rayleigh limit when its net charge reaches a value $Q_{R}$ that is given by $[1,32]$

$$
Q_{R}=8 \pi\left(\varepsilon_{0} \gamma R^{3}\right)^{1 / 2}
$$

where $\varepsilon_{0}$ is the permittivity of the vacuum, and $\gamma$ is the surface tension. In the ESI-MS literature the relationship between $Q_{R}$ and droplet stability is often treated somewhat casually, using statements such as [33] "[it was] found that as the solvent evaporated the density of charges on the droplet surface would increase to a critical value, now known as the 'Rayleigh limit', at which Coulomb repulsion would overcome surface tension. The resulting instability would cause the droplet to break up into a plurality of offspring droplets" (this quotation is meant to represent a typical example; it is not to be construed as a criticism of reference [33]). Statements of this type will prompt a number of questions, especially from readers less familiar with theoretical aspects of the ESI process. (1) How exactly can the interplay of Coulomb forces and surface tension be interpreted, considering that the two factors do not even share the same physical units? (2) What is the reason for the generally observed asymmetry in product size, considering that Rayleigh's mechanism [32] should favor the formation of two equally sized fragments $[9,18,34,35]$ ? (3) What are the consequences of offspring droplets being ejected from the tip of extended protrusions, instead of being emitted from the surface of a more or less spherical parent droplet? (4) How is it possible for these protrusions to form, considering that surface tension tends to counteract any deviations from spherical shape? (5) Why does disintegration occur in bursts that produce several offspring droplets, instead of just a single one? These and other aspects may be addressed through detailed computational and theoretical approaches, where the intricate fluid dynamics of highly charged liquids are modeled from first principles. However, high-level endeavors of this kind do not necessarily provide an intuitive understanding. The current work pursues a different strategy that is based on a very simple "toy model." Nonetheless, the framework used here can provide qualitative answers to the questions outlined above.

\section{Background}

For modeling the breakup of highly charged solvent droplets, we consider these systems to consist of a perfectly conducting continuum fluid, in thermal con- tact with their surroundings. Levitation experiments $[36,37]$ have shown that the time scale of the actual disintegration events is short compared with solvent evaporation. For reasons of simplicity, therefore, evaporation is not explicitly included in our model unless noted otherwise.

The fissility $f$ of a droplet is defined as $f=E_{C} / 2 E_{S}$ $[6,38,39]$ where $E_{C}$ is the Coulomb (electrostatic) energy of a sphere with net surface charge $Q$ and radius $R$

$$
E_{C}=\frac{1}{8 \pi \varepsilon_{0}} \frac{Q^{2}}{R}
$$

and $E_{S}$ is the corresponding surface energy (area $\times$ surface tension)

$$
E_{S}=4 \pi \gamma R^{2}
$$

Droplets tend to be round because a sphere represents the shape with the lowest surface area (and hence the lowest $E_{S}$ ) for a given volume. When expressing the droplet charge relative to $Q_{R}$ using the factor $q=Q / Q_{R}$ the fissility becomes

$$
f=q^{2}
$$

such that $f=1$ for $Q=Q_{R}(q=1)$. For analyzing the fragmentation process it is useful to introduce a reaction coordinate $d$. Energy profiles can be obtained by plotting the energy difference

$$
\Delta E=E(d)-E_{\text {parent }}
$$

where $E_{\text {parent }}$ is the sum of the parent droplet electrostatic and surface energies, and $E(d)$ is the corresponding energy sum for a particular configuration en route to offspring droplet formation. An important parameter for the kinetics of the process is the height of the energy barrier $\Delta E_{t s}$ separating reactants and products

$$
\Delta E_{t s}=E\left(d_{t s}\right)-E_{\text {parent }}
$$

where $E\left(d_{t s}\right)$ is the transition-state energy, also referred to as "saddle point energy" in the fission literature [40]. Considerable deformation of the system at $d_{t s}$ into a non-spherical shape results in a high surface energy. At the same time, the emerging disintegration products are in close proximity, such that the Coulomb energy remains high. As a result, $d_{t s}$ represents the most energetically unfavorable configuration. For $\Delta E_{t s}>0$ fission may occur as thermally activated barrier crossing, characterized by a rate constant $k$ that follows an Arrhenius-type expression [41]

$$
k=A \exp \left(-\Delta E_{t s} / R T\right)
$$

with a pre-exponential factor $A$ and the gas constant $R$. Barrier crossing will be facilitated under conditions of 
elevated temperature $T$. If different fragmentation channels compete with each other (leading to fragments of different size and charge) the extent to which each channel becomes populated depends chiefly on the corresponding value of $\Delta E_{t s}$. Channels with low $\Delta E_{t s}$ will be favored over those with higher barriers. After the system has crossed the transition-state, the emerging fragments may still remain connected by a liquid bridge. Because the liquid is conductive, charge migration can occur up to the scission point $\left(d_{s p}\right)$ at which the connection ruptures.

For deriving eq 1, Rayleigh [32] assumed that fragmentation is triggered by shape oscillations. Droplet deformations were described in terms of spherical harmonics, and he concluded that the fragmentation channel having the lowest $\Delta E_{t s}$ is associated with quadrupole (prolate-oblate [9]) oscillations, whereas higher order deformations are less effective at mediating disintegration [42]. For a neutral droplet $(f=0)$ disintegration is highly endothermic due to the increase in total surface area. Fission gradually becomes more favorable as $f$ increases from zero to unity. In this range the surface enlargement is more and more offset by a reduction in Coulomb energy due to charge separation. In Rayleigh's model, fission under these intermediate conditions can be exo- or endothermic, and product formation involves barrier crossing as discussed above. At $f=1$ the barrier height becomes zero such that disintegration takes place spontaneously in a downhill fashion [39, 43]. Droplets with $f>1$ are even more highly labile [35]. The concepts outlined here for a charged liquid droplet can similarly be applied to metal clusters [38] and atomic nuclei [44].

\section{The Model}

Almost 100 years after Rayleigh's seminal paper [32], Ryce and Wyman developed a "two sphere model" for the breakup of charged droplets $[18,45]$. Variations of the model have recently been applied for studying the fragmentation of gas-phase protein complexes [46-48]. Droplet disintegration according to Ryce and Wyman $[18,45]$ can be described as a budding process, where a fully developed offspring droplet emerges and subsequently separates from its parent. The two droplets remain connected by a conductive filament until the system reaches the scission point. Within the two sphere model it is assumed that this filament has a zero surface energy and carries no charge. This is an obvious problem, because it is known today that offspring droplets are emitted from elongated protrusions, and that the protrusion diameter is comparable to that of the ejected droplet [14-17]. Thus, the assumption of an infinitely thin connection is not tenable.

This work develops a simple framework for the disintegration of charged droplets that addresses the limitations of the two sphere model. A central feature of Ryce and Wyman's work [18, 45] that is retained here is the use of charged spheres, but the current study employs multi-bead assemblies. Determining charge distributions, as well as Coulomb and surface energies for irregularly shaped objects (such as droplets during fragmentation) can be a daunting task. For structures consisting of spheres, however, these calculations are straightforward. Accordingly, a key element of our model is the approximation of solvent filaments as strings of closely packed beads. Considering that these protrusions can exhibit considerable thickness undulations [29, 49], modeling filaments as bead strings appears to be a reasonable approach for gaining qualitative insights into the process.

We consider a parent droplet with radius $R_{0}$ and charge $Q_{0}$. At some reaction coordinate value $d<d_{s p}$ a liquid filament has emerged from the droplet. This entire structure is described as a linear chain of $n$ beads. Bead 1 corresponds to the residual parent droplet with radius $R_{1}\left(R_{1}<R_{0}\right)$. The protrusion is represented as a chain of $(n-1)$ beads, all of which have the same radius $R_{2}\left(R_{2}<R_{1}\right)$. It is convenient to express all radii in dimensionless units $\left(r_{i}=R_{i} / R_{0}\right.$ with $\left.i=0,1,2\right)$ such that $r_{0}=1$, and

$$
r_{1}=\left(1-n r_{2}^{3}\right)^{1 / 3}
$$

as illustrated in Figure 1. Growth of the filament may be envisioned in different ways. For example, one can consider a mode whereby successive additional beads emerge from the residual parent and become part of the protrusion. This process is modeled as a stepwise increase of $n$ at constant $r_{2}$. Other types of growth processes will also be considered. The total length of the system, measured as the midpoint distance between the first and the last bead, represents a suitable reaction coordinate $d$. For $n>1$ the value of the $d$ is

$$
d=r_{1}+(2 n-3) r_{2}
$$

This expression holds as long as all beads are attached to each other. Progeny droplet formation at the scission point $d_{s p}$ corresponds to release of the terminal bead $n$. This implies that for $d>d_{s p}$ the value of the reaction coordinate is given by the right hand side of (eq 9), plus and additional term that describes the edge-to-edge distance of beads $n$ and $(n-1)$. For $n=1$ the reaction coordinate value is taken as $d=1$. Formation of a filament, followed by progeny droplet emission within our "multi-sphere model" are schematically depicted in Figure 2.

For determining the energy profile of the reaction (eq 5) one has to calculate the difference in Coulomb and surface energies (eqs 2,3) between the parent droplet and any of the configurations leading to the fragmented system (Figure 2). In addition, the mutual electrostatic repulsion among the beads has to be taken into account. Accordingly, if $Q_{i}$ is the charge on bead $i$, and $R_{i j}$ is the midpoint distance between beads $i$ and $j$ it can be stated that 


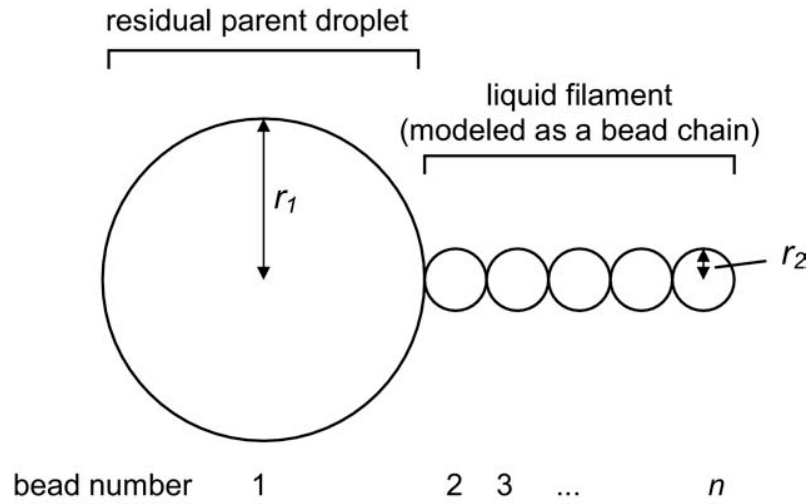

Figure 1. Depiction of a charged droplet that is attached to a liquid filament within the model used here. The filament is described as a chain of beads. The radius of the residual parent droplet, $r_{1}$, and that of the filament beads, $r_{2}$, are indicated. Also illustrated is the bead numbering used throughout this work.

$$
\begin{aligned}
\Delta E= & \frac{1}{8 \pi \varepsilon_{0}}\left(\frac{Q_{1}^{2}}{R_{1}}+\sum_{i=2}^{n} \frac{Q_{i}^{2}}{R_{2}}\right)+\frac{1}{4 \pi \varepsilon_{0}} \sum_{i=1}^{n-1} \sum_{j=i+1}^{n} \frac{Q_{i} Q_{j}}{R_{i j}} \\
& +4 \pi \gamma\left(R_{1}^{2}+(n-1) R_{2}^{2}\right)-\left(\frac{Q_{0}^{2}}{8 \pi \varepsilon_{0} R_{0}}+4 \pi \gamma R_{0}^{2}\right)
\end{aligned}
$$

This equation is best dealt with by using dimensionless variables. For this purpose all charges are expressed relative to the Rayleigh charge of the parent droplet.

$$
q_{i}=Q_{i} /\left[8 \pi\left(\varepsilon_{0} \gamma R_{0}^{3}\right)^{1 / 2}\right]
$$

In addition, all energy differences are reported relative to the surface energy of the parent droplet

$$
\Delta e=\frac{\Delta E}{4 \pi \gamma R_{0}^{2}}
$$

Dimensionless bead radii $\left(r_{1}, r_{2}\right)$ have already been introduced above. With these transformations the energy profile of the reaction (eq 10) becomes

$$
\Delta e=\Delta e_{C}\left(r_{2}, q_{0}, n\right)+\Delta e_{S}\left(r_{2}, n\right)
$$

with the normalized Coulomb energy change

$$
\Delta e_{C}\left(r_{2}, q_{0}, n\right)=\frac{2 q_{1}^{2}}{r_{1}}+\sum_{i=2}^{n} \frac{2 q_{i}^{2}}{r_{2}}-2 q_{0}^{2}+\sum_{i=1}^{n-1} \sum_{j=i+1}^{n} \frac{4 q_{i} q_{j}}{r_{i j}}
$$

the normalized surface energy change

$$
\Delta e_{S}\left(r_{2}, n\right)=r_{1}^{2}+(n-1) r_{2}^{2}-1
$$

and the activation barrier height $\Delta e_{t s}$. As implied by the notation used in eqs $13-15, \Delta e$ depends on only three independent parameters as long as all beads are attached to each other, namely $r_{2}, q_{0}$, and $n$. The inter- bead distances $r_{i j}$ are obtained from geometric considerations (Figure 2). Conservation of volume allows $r_{1}$ to be eliminated according to eq 8 . Similarly, conservation of charge implies that $q_{0}=\sum_{i=1}^{n} q_{i}$, where the $q_{i}$ values on the individual beads correspond to the lowest possible value of $\Delta e_{C}$ in eq 14. For $n=2$ the charge distribution can be determined analytically [45], but for $n>2$ such a strategy quickly becomes impractical. The current work therefore uses a numerical optimization procedure for determining the $q_{i}$ values on beads $1, \ldots$, $n$ that correspond to the global minimum of $\Delta e_{C}$. For $d>d_{s p}$ the terminal bead is separated from the rest of the system. From this point on, $n$ remains constant, being replaced by $d$ as an independent parameter. The distance separating beads $n$ and $(n-1)$ has to be taken into

(a)

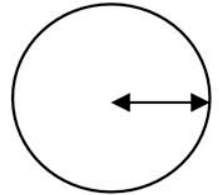

(b)

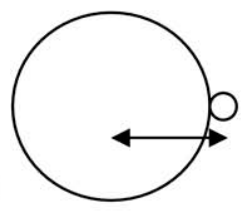

(c)

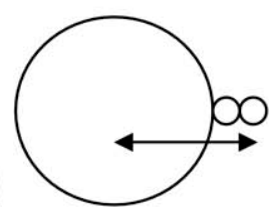

(d)

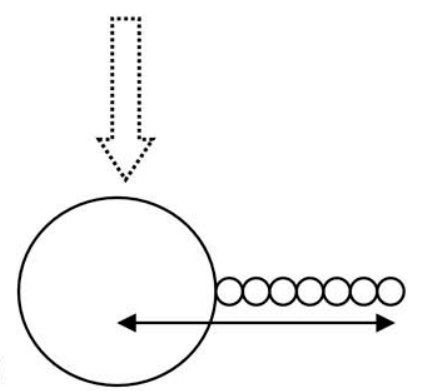

(e)

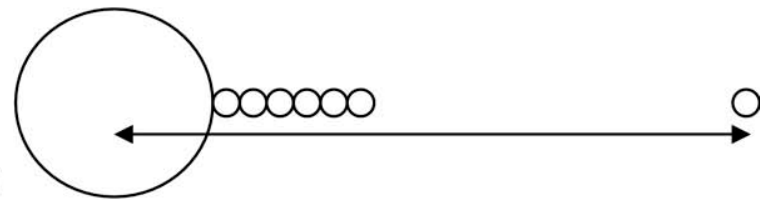

Figure 2. Droplet configurations along a possible fragmentation pathway. (a) Intact parent droplet; (b) Onset of protrusion formation; (c) protrusion growth; (d) Several steps later the liquid filament has fully formed; (e) The system has passed the scission point and a progeny droplet (terminal bead) has been ejected. Double-headed arrows represent the value of the reaction coordinate $d$ at each stage, representing the overall length of the system. 
account for determining distance values $r_{i n}$. Finally, when using the dimensionless coordinates introduced above, eq 4 for calculating droplet fissilities has to be modified according to

$$
f_{i}=q_{i}^{2} r_{i}^{-3}
$$

The data discussed in the following sections were obtained using a FORTRAN program that was written based on eqs $8-16$. Charge values $q_{i}$ were determined using the IMSL routine UMINF (Visual Numerics, Houston, TX).

\section{Results and Discussion}

The multi-bead model outlined in the previous sections (Figures 1,2) was used for exploring the energetics of progeny droplet emission from the tip of a liquid filament that is attached to the residual parent droplet. Droplet emission is preceded by formation of the filament.

\section{Charge Distributions}

Before discussing the droplet disintegration processes itself, it is instructive to examine energy-minimized spatial charge distributions for two different bead assemblies, initially focusing on a parent droplet at the Rayleigh limit $\left(q_{0}=1\right)$. We first consider an arrangement that is not meant to resemble an intermediate of the fragmentation process, namely one where the parent droplet has been distorted into a chain of equally sized beads $(n=11)$. The radius of each bead in this case is $r_{2}=11^{-1 / 3}=0.4496$. Electrostatic repulsion leads to charge accumulation on the two terminal segments $\left(q_{1}=q_{11} \approx 0.15\right)$, whereas the beads in the center of the chain carry lower charges around 0.077 (Figure 3a). Close inspection reveals local charge minima in positions 2 and 10, caused by electrostatic repulsion from the highly charged neighboring termini. Two conclusions regarding the energy-minimized charge distribution on liquid filaments can be drawn (1) charge accumulation occurs at the termini and (2), an aboveaverage charge on one segment induces charge depletion in adjacent sections. Another scenario is depicted in Figure $3 b$, where a residual parent droplet (bead 1) is attached to a protruding filament consisting of ten small beads with $r_{2}=0.15$. As expected based on its much larger capacitance, the residual parent accommodates most of the total charge. Repulsion leads to considerable depletion in adjacent segments, with bead 2 carrying a charge close to zero. Bead 11 at the end of the filament represents the second most highly charged segment. Already, from the data in Figure $3 b$ it is apparent that ejection of the terminal segment as a progeny droplet will reduce the charge of the overall system by a relatively large amount (6\% for the parameter set used here), despite its very small-volume frac-

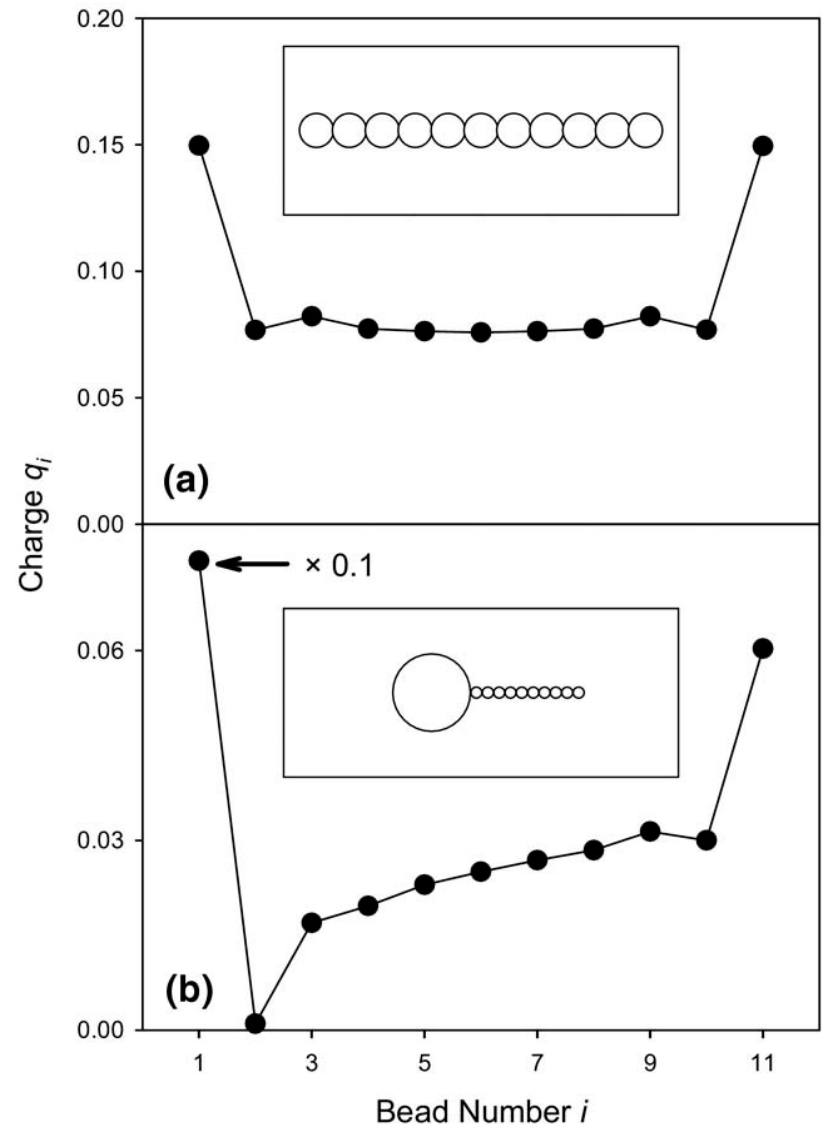

Figure 3. Charge values $q_{i}$ for two different bead assemblies of the same total charge $q_{0}=1$. (a) Linear string of 11 beads, as shown in the inset. (b) Large residual parent droplet attached to a 10 bead-filament. Note that the first data point in panel $\mathbf{b}$ has been scaled down by a factor of 10 .

tion of $0.3 \%$. This feature represents one of the hallmarks of the ESI process [13].

\section{Droplet Breakup after Filament Growth at Constant Diameter}

Several modes of filament growth can be envisioned within the framework developed here. We will first consider the formation of a protrusion at constant diameter, modeled as a stepwise increase in the number of beads $n$ at constant $r_{2}$. The sequence corresponding to $n=1, \ldots, 11$, with $r_{2}=0.15$ and $q_{0}=1$ will serve as an example. It is assumed that the scission point $d_{s p}$ is reached once the eleventh bead has formed, corresponding to the scenario depicted in Figure 3b. Several system properties associated with this process are plotted as a function of the reaction coordinate $d$ in Figure 4. Filament growth causes a pronounced increase in surface energy $\Delta e_{S}$ as the system deviates more and more from its initial spherical shape. At the same time, formation of the protrusion allows charge to spread out, resulting in a decrease in Coulomb energy $\Delta e_{C}$ (Figure 4a). The increase in $\Delta e_{S}$ ends with the onset of progeny droplet (bead 11) ejection at $d_{s p}$. In contrast, $\Delta e_{C}$ contin- 


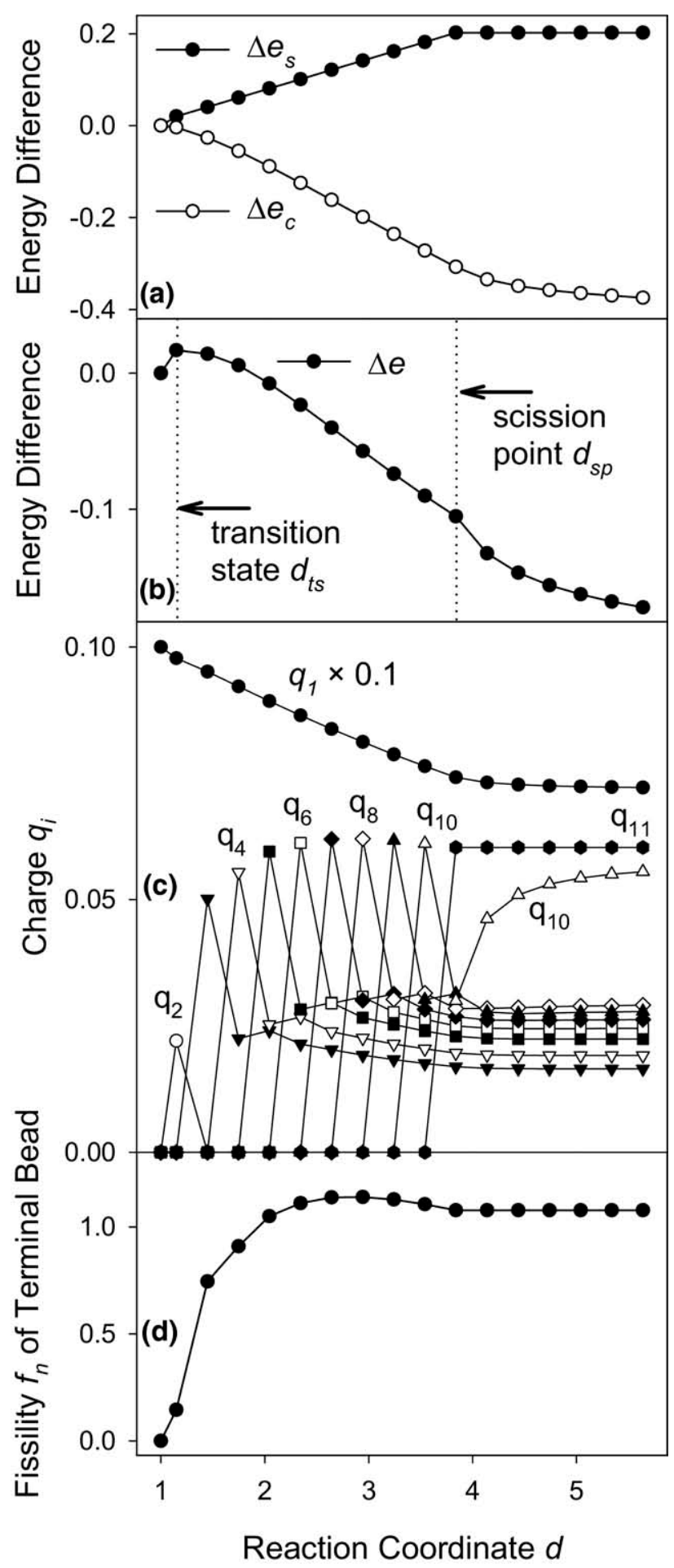

Figure 4. Reaction coordinate profiles of various system properties for the fission scenario outlined in Figure 2, i.e., filament growth at constant diameter, followed by progeny droplet ejection. Parameters used: $r_{2}=0.15, q_{0}=1$, and $n=1, \ldots, 11$. (a) Difference in Coulomb energy $\Delta e_{C}$ and surface energy $\Delta e_{S}$. (b) Overall energy difference $\Delta e$, representing the sum of the two contributions in panel a. Also shown are the location of the transition-state $d_{t s}$, and the scission point $d_{s p}$. (c) Charge $q_{i}$ on each of the beads during filament growth, and after progeny droplet ejection. Labels for $q_{3}, q_{5}, q_{7}$, and $q_{9}$ have been omitted to reduce cluttering. (d) Fissility $f_{n}$ of the terminal filament bead. ues to drop while the progeny droplet separates further and further from the newly formed end of the protrusion, driven by electrostatic repulsion.

Inspection of the overall energy profile (Figure 4c) shows that the first step of filament growth, i.e., formation of bead 2, is associated with an energy barrier $\Delta e_{t s}>0$. In contrast, all subsequent steps proceed energetically downhill, leading to the exothermic formation of a long protrusion all the way to $d_{s p}$. In other words, an initial small bulge on the surface of the parent droplet (at $n=2$ ) acts as nucleus for the spontaneous growth of a long protrusion. The driving force of the process for $n>2$ is the decrease in electrostatic energy, which overcompensates for the unfavorable gain in surface energy. The energetic downhill nature of filament growth makes it understandable why it is possible for highly charged droplets to form very long appendices [14-17], resulting in shapes reminiscent of those that might be produced by mechanically stretching a viscous object (bubble gum pulling analogy).

Figure 4c illustrates how the charge distribution within the system changes during filament growth and after progeny ejection. As expected based on Figure 3b, a major fraction of the total charge remains on bead 1 throughout the whole process. Yet, gradual increase of the filament surface area allows the displacement of more and more charge from the residual parent to the filament beads ( $q_{1}$ curve in Figure $\left.4 c\right)$. The terminal segment always carries a larger charge than all the preceding filament beads. This implies a dramatic drop in the charge $q_{n-1}$ every time a new bead $n$ is formed, giving rise to the characteristic sawtooth pattern for $q_{2}$ to $q_{10}$ in Figure $4 c$. Ejection of bead 11 at $d_{s p}$ is accompanied by a marked increase of $q_{10}$, as bead 10 takes over as the new terminus. As we will discuss later, this implies that the system is now poised for the ejection of additional progeny droplets. Another interesting feature is that the charge residing on the terminal bead during growth of the protrusion increases for the first few elongation steps, and then saturates after ca. six beads have been formed. Close inspection of Figure $4 \mathrm{c}$ even reveals a slight decrease past the eighth elongation step, which is based on a "dilution" effect as the total charge spreads over the rapidly increasing surface area.

Throughout the process one can assign a fissility value $f_{n}$ to the terminal bead according to eq 16 (Figure $4 \mathrm{~d}$ ). It is more common to discuss $f$ values for isolated droplets and not for segments within a larger liquid structure. Nonetheless, the data in Figure $4 \mathrm{~d}$ represent a measure of the "electrostatic stress" experienced by the chain terminus. The particular scenario considered here leads to an unstable progeny droplet with a fissility slightly larger than unity. Under such conditions the high electric field at the tip of the protrusion might result in ion emission even before progeny droplet formation, assuming the presence of analytes that are amenable to IEM ionization. 


\section{Activation Barrier Effects on Progeny Droplet Size}

As noted in the Background section, fragmentation channels with low activation barriers will be favored over those with larger $\Delta e_{t s}$ values. This has major implications for the size of the progeny droplets being formed. To determine which barrier heights are energetically accessible, one has to consider the magnitude of the spontaneous energy fluctuations that the parent droplet undergoes as it interacts with its surroundings. Expressed on a normalized scale, the standard deviation $\sigma_{e}$ of the droplet internal energy is [50]

$$
\sigma_{e}=\frac{\sqrt{k T^{2} C_{V}}}{4 \pi \gamma R_{0}^{2}}
$$

where $k$ is Boltzmann's constant and $C_{V}$ is the heat capacity. For $R_{0}=0.5 \mu \mathrm{m}$ and a temperature of $T=370$ $\mathrm{K}$ this corresponds to $\sigma_{e} \approx 0.01$. Thus, micrometer-sized parent droplets that are typically encountered during the early stages of ESI [1] can readily overcome energy barriers of $\Delta e_{t s} \approx 0.01$ in the heated environment of a typical ion source. Fragmentation channels associated with $\Delta e_{t s}$ values that considerably exceed 0.01 will not become strongly populated.

Readers are reminded that the progeny droplet size within our model is determined by the radius of the filament beads, a feature that is supported by previous studies [14-17]. For the case of filament growth at constant radius, Figure 5a illustrates how the overall energy profile $\Delta e$ depends on $r_{2}$. Note that these data are plotted versus $n$, and not versus the reaction coordinate $d$. Bead radii of 0.2 and larger are associated with a barrier at onset of filament growth (formation of bead 2 , dotted line in Figure 5a). Barrier heights $\Delta e_{t s}$ of 0.03 to 0.08 are encountered for $r_{2}$ between 0.2 and 0.4 are (Figure 5b). Even larger $\Delta e_{t s}$ values are found for $r_{2}>$ 0.4 (data not shown). These barriers considerably exceed the thermally accessible range of $\Delta e \approx 0.01$, indicated by the dashed line in Figure $5 \mathrm{~b}$. Therefore, filament and progeny droplet formation with $r_{2}$ values of ca. 0.2 and larger is energetically disfavored for the growth mode considered here.

A different situation is encountered for thinner filaments that give rise to correspondingly smaller progeny droplets. The transition-state is shifted to $n=3$ for $r_{2}$ values of 0.05 and 0.1 . More importantly, the barrier heights under these conditions are considerably lower due to the smaller surface area changes involved. The value of $\Delta e_{t s}$ for $r_{2}=0.1$ is slightly below 0.01 . An almost barrier-free transition $\left(\Delta e_{t s}=0.003\right)$ is encountered for $r_{2}=0.05$. These considerations show that the formation of very small progeny droplets (resulting from thin filaments) is kinetically favored, thus providing a simple explanation for the prevalence of asymmetric fission events during ESI.

A complicating factor that has to be discussed is the following: The onset of filament formation is kinetically

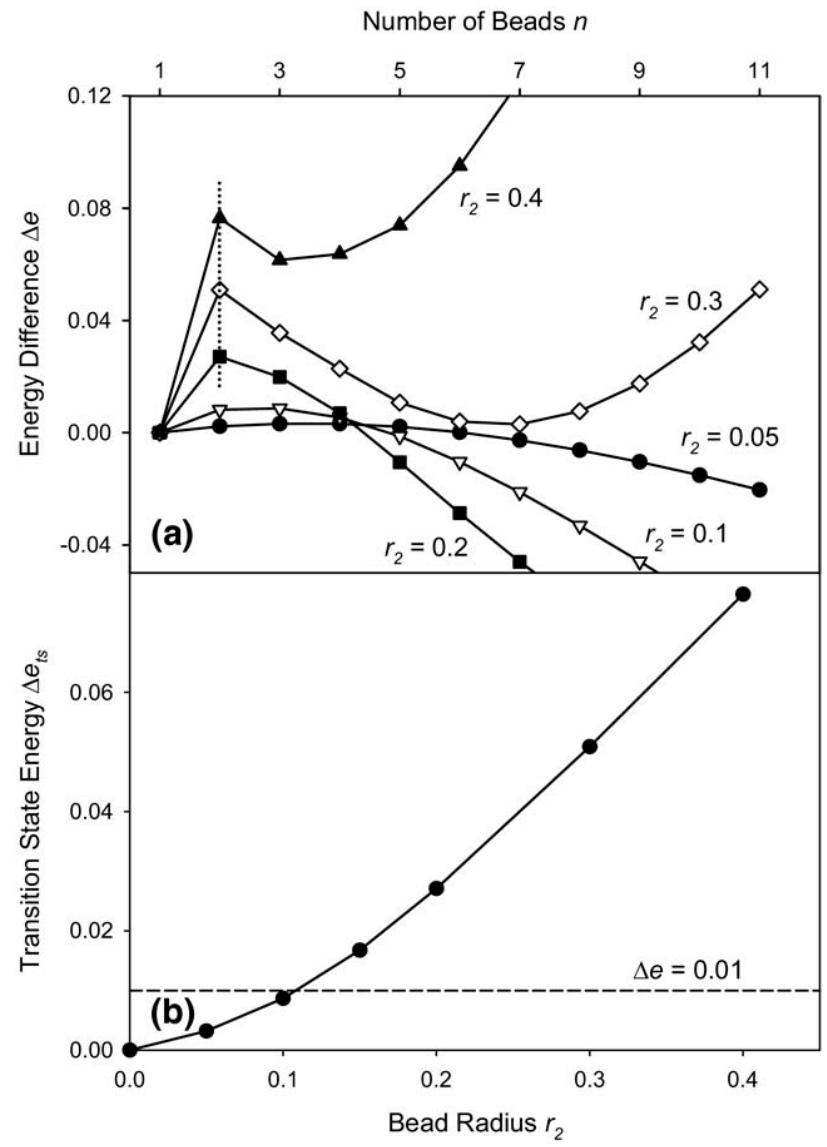

Figure 5. (a) Overall energy difference $\Delta e$ for filament growth at constant $r_{2}$ for $q_{0}=1$. The dotted line marks the transitionstate at $n=2$ for datasets with $r_{2}=0.2$ to $r_{2}=0.4$. Progeny droplet ejection is not considered here, but might occur at any point where $n>1$. (b) Transition-state energy $\Delta e_{t s}$ for the data from panel $\mathbf{a}$. The dashed line in panel $\mathbf{b}$ indicates the threshold level of $\Delta e=0.01$.

favored for small values of $r_{2}$, however, this situation tends to result in unrealistically high fissility values if the growth process continues to large values of $n$. For example, the $r_{2}=0.05$ dataset in Figure $5 \mathrm{~b}$ reaches $f_{n}=$ 2.1 for $n=11$. Theoretical studies support the idea that progeny droplets may be charged close to the Rayleigh limit under some conditions [51]. However, supercharging the terminal segment all the way to $f=2.1$ seems unrealistic because secondary disintegration would likely take place long before this point. Chain growth in this particular case would not be expected to proceed far beyond $n=5(d=1.35)$, where $f_{n}$ reaches a value of unity. In summary, based on the parameter space explored so far, our model predicts the formation of very thin but short liquid protrusions as intermediates of the droplet disintegration process. Experimental observations, on the other hand, have revealed the presence of thin and long filaments [15-17]. In the following section we will examine how this apparent inconsistency can be resolved. 


\section{Alternative Modes of Filament Growth}

The previous discussion focused on only one particular mode of filament growth, namely elongation at constant diameter. We will now examine growth processes that involve changes in both $n$ and $r_{2}$. It has to be tested if excessive fissilities of the terminal segment for large $n$ can be avoided in this way, and if the prediction of highly asymmetric fission remains valid.

The considerable energy barriers seen in Figure 5 for $r_{2}>0.1$ are caused by the formation of a large filament bead at the $n=1 \rightarrow n=2$ transition. Such a budding scenario may not be the most realistic description for the onset of filament growth. A more likely mechanism is the initial formation of a slender microprotrusion [9, 10], which then grows long and thick. Such a scenario is shown by the energy profile data in Figure 6, where a microprotrusion is formed by filament growth up to $n=5$ at a constant (but very small) value of $r_{2}=0.05$. The following three steps are modeled as an increase in bead radius to $r_{2}=0.1,0.15$, and 0.2 , at a constant value of $n=5$. Raising $r_{2}$ in this way can occur spontaneously because it is associated with a continuous energy decrease. A further increase in $r_{2}$ at constant $n$, however, is energetically uphill and quickly reaches $\Delta e$ values that are thermally inaccessible (open symbols in Figure 6). Alternatively, the system can revert to elongation at constant diameter once $r_{2}=0.2$ has been reached. Commencing the growth process through a stepwise increase in $n$ at this point allows further energetic relaxation in a downhill fashion (Figure 6, solid symbols).

This example demonstrates how formation of a long and fairly thin filament $\left(n=11, d=4.8, r_{2}=0.2\right)$ can occur spontaneously if growth proceeds through an initial microprotrusion, followed by an increase in radius, and subsequent extension at constant $r_{2}$. The activation barrier in this scenario is close to zero $\left(\Delta e_{t s}=\right.$

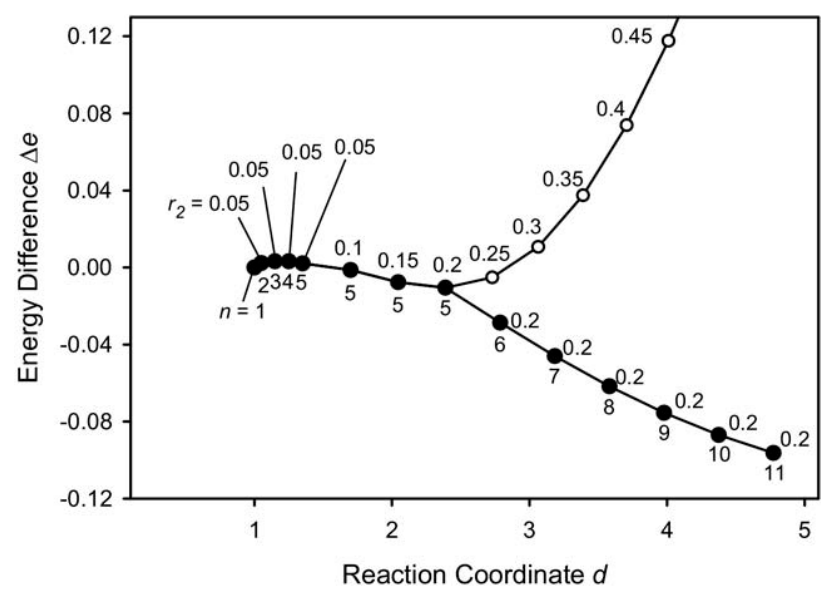

Figure 6. Overall energy difference $\Delta e$ for filament growth through a combination of bead insertion and diameter increase at $q_{0}=1$. Values of $r_{2}$ and $n$ are denoted above and below, respectively, of the data points. Progeny droplet ejection is not considered. For details see text.
0.003). Formation of the same filament at constant $r_{2}$ would be virtually impossible due to the presence of a 10-fold higher barrier (Figure 5). Notably, the pathway highlighted by the solid symbols in Figure 6 avoids excessive fissilities for the terminal bead. After peaking at $f_{n}=1.1$ for $d=1.7$, the fissility drops to 0.76 for the fully formed filament. At the same time, asymmetric droplet fission (with $r_{2} \leq 0.2$ ) remains strongly favored in this modified scenario.

\section{Effects of Parent Droplet Charge}

Early experiments suggested that disintegration can already occur for droplets that are charged to only $70 \%$ to $80 \%$ of the Rayleigh limit $[15,52]$. However, more recent and accurate data have shown that breakup only happens for systems very close to $Q=Q_{R}[36,37,53]$. Energy profiles were calculated in this study to explore the effects of parent droplet charge on the fission process. Out of the many possible fragmentation pathways, we chose the optimized scenario of Figure 6 (solid symbols), which is associated with the lowest energy barrier. It was assumed that the scission point corresponds to $n=11$ at $r_{2}=0.2$ (Figure 7). Under these conditions, fragmentation for a parent droplet at $80 \%$ of the Rayleigh limit $\left(q_{0}=0.8\right)$ is not feasible because it would involve crossing of a thermally inaccessible barrier $\left(\Delta e_{t s} \gg 0.01\right)$. The process becomes more favorable for $q_{0}=0.9$, but even under these conditions a sizeable barrier persists. In contrast, fragmentation at the Rayleigh limit $\left(q_{0}=1\right)$ occurs with an activation barrier close to zero and in a highly exothermic fashion. For more highly charged droplets $\left(q_{0}=1.5, q_{0}=2\right)$ the process is even more favorable. Very similar conclusions are reached when testing other possible fragmentation pathways (data not shown). Thus, our model confirms the experimental finding $[36,37,53]$ that the Rayleigh limit $\left(q_{0}=1.0\right)$ represents a fundamental threshold for the behavior of highly charged droplets, below which fission is unlikely to occur. This agreement is remarkable, considering that the fragmentation mechanism discussed here (growth of a multi-bead filament, followed by progeny droplet emission) is completely different from that stipulated by Rayleigh [32].

\section{Emission of Multiple Progeny Droplets}

Let us now consider a parent droplet with $q_{0}=1$ that has reached the scission point by forming an extended filament. Emission of the terminal segment $n$ reduces the charge on the residual system [(beads 1 to $(n-1)$ ] down to $1-q_{n}$. A spherical parent droplet carrying such a sub-Rayleigh charge would be unlikely to undergo disintegration, as discussed in the previous paragraph. But the situation is different for the case considered here; after ejection of bead $n$ a filament remains attached to the residual parent, such that a subsequent emission event can occur without requiring barrier 


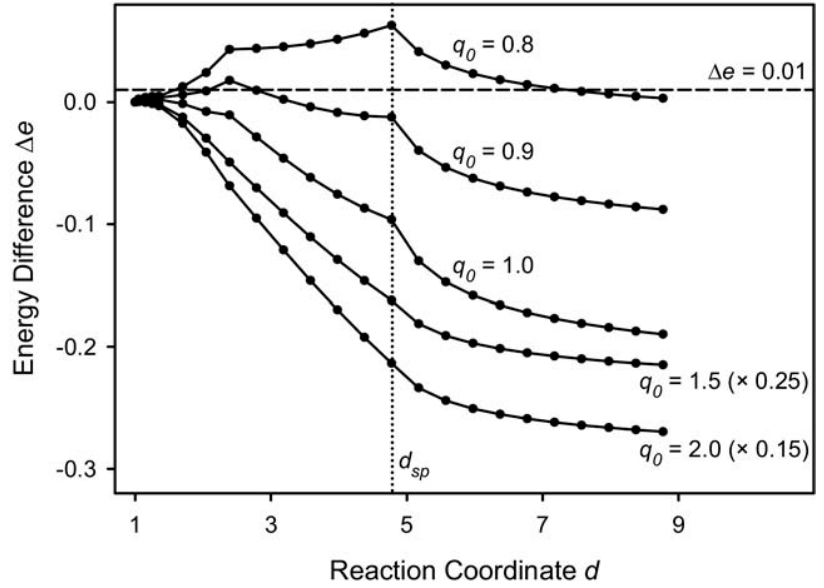

Figure 7. Overall energy difference $\Delta e$ for filament growth following the mechanism indicated by the solid symbols in Figure 6. Data are depicted for different parent droplet charge values $q_{0}$. Progeny droplet emission occurs at the scission point $d_{s p}$ (vertical dotted line). The dashed horizontal line indicates the threshold level of $\Delta e=0.01$. Data for $q_{0}=1.5$ and $q_{0}=2.0$ have been rescaled as indicated.

crossing. From the data in Figure 4c we know that ejection of the terminal bead $n$ is followed by charge accumulation on the new terminus $(n-1)$. Thus, bead $(n-1)$ can now be ejected as a second progeny droplet, and $(n-2)$ becomes the new terminus. This may be followed by ejection of bead $(n-2)$, and so on. In this way a series of progeny droplets may be produced, each of which carries with it a substantial amount of charge but only very little volume. This stepwise charge depletion gradually reduces the Coulombic repulsion within the system to a point where collapse of the filament becomes energetically favored. Progeny droplet emission stops once the residual system has returned to a spherical shape.

The energetics of such a sequence are depicted in Figure 8, arbitrarily assuming that $r_{2}=0.2$ for all values of $n$. We assume that the system has reached its scission point at $n=11$. The temporal development from this point on is associated with either an increase or a decrease of the reaction coordinate $d$. Values $d>d_{s p}$ correspond to emission of a progeny droplet. In contrast, values for $d<d_{s p}$ represent collapse of the system, where the protrusion shrinks by merging of filament beads with the residual parent. Figure 8a represents the situation where $n=11$ and $q_{0}=1.0$. Filament collapse from $d_{s p}$ is energetically uphill, whereas progeny droplet emission, with $q_{11}=0.078$, is highly favorable. Panel $\mathrm{B}$ represents the residual system, which now carries a charge of $1.0-0.078=0.922$. Droplet emission under these conditions remains highly favored, and the charge on the emitted bead is $q_{10}=0.073$. In Figure $8 \mathrm{c}$ the residual system now carries a charge of $0.922-0.073=$ 0.849 . Inspection of the $\Delta e$ profile reveals that collapse of the filament would be an exothermic process, but this would require crossing of an energy barrier. Thus, emission of a third progeny droplet with $q_{9}=0.070$ remains the more favorable alternative. For the subse-

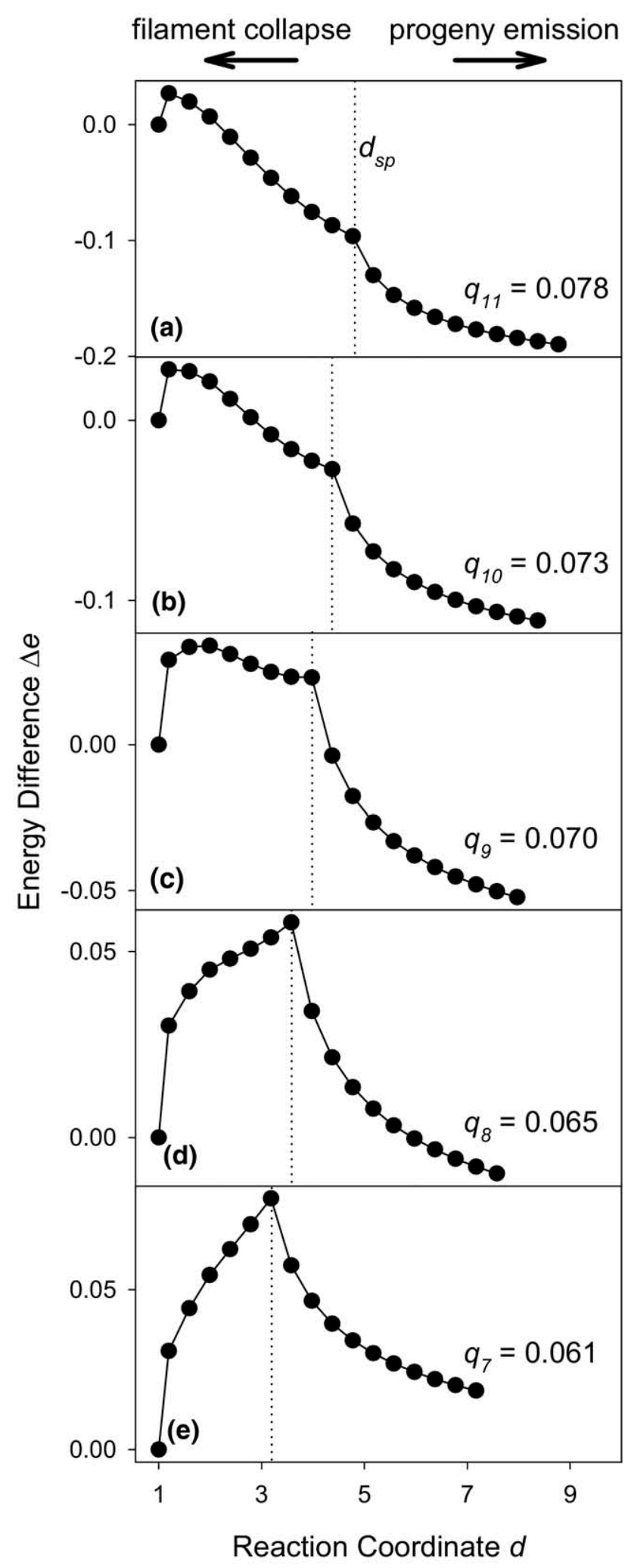

Figure 8. Energy profiles for systems that undergo either progeny droplet ejection or filament collapse at successively lower charge and successively shorter filaments $\left(r_{2}=0.2\right)$. The charge emitted with each progeny droplet and the corresponding bead number are indicated in (a)-(e). Dotted lines indicate the location of the scission point in each panel. For further explanations see text. 
quent scenario of Figure $8 \mathrm{~d}$ the system is now severely depleted of charge, with a total $q$ value of only $0.849-$ $0.070=0.779$. Under these conditions both filament collapse and progeny emission represent viable options, since both are energetically downhill. Should emission of droplet 8 occur $\left(q_{8}=0.065\right)$, the remainder of the system would be left with a charge of only $0.779-0.065=0.71$. At this point, the potential energy gradient for collapse is comparable to that for emission of droplet 7 (Figure 8e). Emission of additional droplets remains a possibility if this sequence is continued, but the driving force for filament collapse increases with each subsequent step (data not shown). Every emitted progeny droplet in our model carries a charge slightly lower than the previous one.

It is seen from the these considerations how the model predicts that a droplet at the Rayleigh limit emits not one, but several progeny droplets. This prediction is consistent with experimental observations $[1,13,15,16]$. The system returns to a spherical shape once charge depletion has occurred to such an extent that maintaining the protrusion is energetically no longer viable. Progeny droplet emission can only commence if solvent evaporation reduces the droplet diameter until it once again reaches the Rayleigh limit.

\section{Conclusions}

Toy models describe complex physical phenomena in simplified ways, nonetheless capturing the essence of the underlying processes. In this study, we examined the interplay of surface energy and Coulombic repulsion that governs the disintegration of highly charged solvent droplets, for example under ESI conditions. Approximating the shapes of distorted droplets as bead assemblies greatly simplifies the energetics of the system. Naturally, using such a basic approach has its limitations. For example, the model has no bearing on previously derived scaling laws $[14,51]$ that describe how the process depends on conductivity, dielectric constant, density, and viscosity. Referring back to the five points raised in the Introduction section, the following conclusions can be reached.

1. Equation 10 provides a basic tool for analyzing the opposing effects of Coulomb repulsion (which favors filament formation and progeny droplet emission), and surface tension (which favors the retention of a spherical parent droplet).

2. Whether or not a certain physicochemical pathway is viable depends chiefly on the height of the corresponding activation energy barrier [41]. Development of a thin liquid filament is associated with a lower barrier than formation of a protrusion with large diameter. This difference in barrier height implies the prevalence of progeny droplets that are much smaller than their parent (Figures 5,6), given that the filament diameter is comparable to that of the emitted droplet(s) [14-17].
3. The fact that progeny droplets are emitted from the tip of long filaments is responsible for the high charge on these droplets. A small droplet that is directly attached to the surface of a spherical parent carries relatively little charge (see data for $q_{2}$ in Figure 4c). Thus, progeny emission directly from the surface of a spherical parent would be an ineffective mechanism for lowering the electrostatic stress of the system. In contrast, a long filament that is attached to the parent will accumulate a considerable amount of charge at its tip ( $q_{11}$ in Figure $4 c$ ). Release of this terminal segment leads to the formation of a much more highly charged progeny droplet.

4. Surface tension generally tends to favor the retention of an overall spherical shape for the system. However, the internal Coulomb repulsion of droplets at the Rayleigh limit is so extreme that the formation of long filaments can occur spontaneously. This is because the shape deformation allows charge to spread over a larger surface area, keeping in mind that the repulsion between two ions is proportional to their inverse distance. Filament formation for droplets at the Rayleigh limit occurs with an activation barrier close to zero (Figure 7). Our model suggests that the most likely mode of growth is the initial formation of a short and thin nucleation filament, followed by an increase in thickness to $r_{2} \approx$ 0.2 , and subsequent growth of the protrusion at constant diameter until the scission point is reached.

5. Emission of several progeny droplets is favored over the emission of a single fragment. This is because once a filament has been formed as launching site for the first progeny droplet, additional droplet emission is an energetic downhill process without barrier crossing. This allows a large fraction of the system charge to be drained before surface tension causes the filament to collapse. After a number of progeny droplets have been emitted the charge depleted system returns to a spherical shape well below the Rayleigh limit (Figure 8).

In summary, the disintegration of charged droplets during ESI is a complex process, and clearly the very basic framework used here cannot provide a highly accurate description of the underlying dynamics. Nonetheless, comparison with previous experimental and theoretical studies reveals that many features are captured surprisingly well.

\section{Acknowledgments}

The author acknowledges support for this work by The University of Western Ontario and the Canada Research Chairs Program.

\section{References}

1. Kebarle, P.; Peschke, M. On the Mechanisms by which the Charged Droplets Produced by Electrospray Lead to Gas Phase Ions. Anal. Chim. Acta 2000, 406, 11-35. 
2. de la Mora, F. J. Electrospray Ionization of Large Multiply Charged Species Proceeds Via Dole's Charged Residue Mechanism. Anal. Chim. Acta 2000, 406, 93-104.

3. Cole, R. B. Some Tenets Pertaining to Electrospray Ionization Mass Spectrometry. J. Mass Spectrom. 2000, 35, 763-772.

4. Van Berkel, G. J.; de la Mora, J. F.; Enke, C. G.; Cole, R. B.; MartinezSanchez, M.; Fenn, J. B. Electrochemical Processes in Electrospray Ionization Mass Spectrometry. J. Mass Spectrom. 2000, 35, 939-952.

5. Constantopoulos, T. L.; Jackson, G. S.; Enke, C. G. Challenges in Achieving a Fundamental Model for ESI. Anal. Chim. Acta 2000, 406, 37-52.

6. Giglio, E.; Gervais, B.; Rangama, J.; Manil, B.; Huber, B. A. Shape Deformations of Surface-Charged Microdroplets. Phys. Rev. E 2008, 77, 036319 .

7. Feng, X.; Bogan, M. J.; Agnes, G. R. Coulomb Fission Event Resolved Progeny Droplet Production from Isolated Evaporating Methanol Droplets. Anal. Chem. 2001, 73, 4499-4507.

8. Tang, K.; Smith, R. D. Theoretical Prediction of Charged Droplet Evaporation and Fission in Electrospray Ionization. Int. J. Mass Spectrom. 1999, 185/186/187, 97-105.

9. Ichiki, K.; Consta, S. Disintegration Mechanisms of Charged Aqueous Nanodroplets Studied by Simulations and Analytical Models. J. Phys. Chem. B 2006, 110, 19168-19175.

10. Marginean, I.; Znamenskiy, V.; Vertes, A. Charge Reduction in Electrosprays: Slender Nanojets as Intermediates. J. Phys. Chem. B 2006, 110, 6397-6404.

11. Nemes, P.; Marginean, I.; Vertes, A. Spraying Mode Effect on Droplet Formation and Ion Chemistry in Electrosprays. Anal. Chem. 2007, 79 , 3105-3116.

12. Storozhev, V. B.; Nikolaev, E. N. Computer Simulations of the Fission Process of Charged Nanometer Droplets. Phil. Mag. 2004, 84, 157-171.

13. Kebarle, P.; Tang, L. From Ions in Solution to Ions in the Gas Phase: The Mechanism of Electrospray Mass Spectrometry. Anal. Chem. 1993, 65, 972A-986A.

14. Gu, W.; Heil, P. E.; Choi, H.; Kim, K. Comprehensive Model for Fine Coulomb Fission of Liquid Droplets Charged to Rayleigh Limit. Appl. Phys. Lett. 2007, 91, 064104

15. Gomez, A.; Tang, K. Charge and Fission of Droplets in Electrostatic Sprays. Phys. Fluids 1994, 6, 404-414.

16. Duft, D.; Achtzehn, T.; Muller, R.; Huber, B. A.; Leisner, T. Coulomb Fission: Rayleigh Jets from Levitated Microdroplets. Nature 2003, 421, 128

17. Li, D.; Marquez, M.; Xia, Y. Capturing Electrified Nanodroplets Under Rayleigh Instability by Coupling Electrospray with a sol-gel Reaction. Chem. Phys. Lett. 2007, 445, 271-275.

18. Ryce, S. A.; Wyman, R. R. Asymmetry in the Electrostatic Dispersion of Liquids. Can. J. Phys. 1964, 42, 2185-2194.

19. Enke, C. G. A Predictive Model for Matrix and Analyte Effects in Electrospray Ionization of Singly-Charged Ionic Analytes. Anal. Chem. 1997, 69, 4885-4893.

20. Cech, N. B.; Enke, C. G. Practical Implication of Some Recent Studies in Electrospray Ionization Fundamentals. Mass Spectrom. Rev. 2001, 20, 362-387.

21. Kuprowski, M. C.; Konermann, L. Signal Response of Coexisting Protein Conformers in Electrospray Mass Spectrometry. Anal. Chem. 2007, 79, 2499-2506.

22. Hogan, C. J.; Biswas, P. Monte Carlo Simulation of Macromolecular Ionization by Nanoelectrospray. J. Am. Soc. Mass Spectrom. 2008, 19, 1098-1107.

23. Dole, M.; Mack, L. L.; Hines, R. L.; Mobley, R. C.; Ferguson, L. D.; Alice, M. B. Molecular Beams of Macro-Ions. J. Chem. Phys. 1968, 49, 22402249.

24. Iribarne, J. V.; Thomson, B. A. On the Evaporation of Small Ions from Charged Droplets. J. Chem. Phys. 1975, 64, 2287-2294.

25. Nguyen, S.; Fenn, J. B. Gas-Phase Ions of Solute Species from Charged Droplets of Solutions. Proc. Natl. Acad. Sci. U.S.A. 2007, 104, 1111-1117.

26. Felitsyn, N.; Peschke, M.; Kebarle, P. Origin and Number of Charges Observed on Multiply-Protonated Native Proteins Produced by ESI. Int. J. Mass Spectrom. Ion Processes 2002, 219, 39-62.

27. Nesatyy, V. J.; Suter, M. J.-F. On the Conformation-Dependent Neutralization Theory and Charging of Individual Proteins and Their Noncovalent Complexes in the Gas Phase. J. Mass Spectrom. 2004, 39, 93-97.
28. Iavarone, A. T.; Williams, E. R. Mechanism of Charging and Supercharging Molecules in Electrospray Ionization. I. Am. Chem. Soc. 2003 $125,2319-2327$.

29. Kaltashov, I. A.; Mohimen, A. Estimates of Protein Surface Area in Solution by Electrospray Ionization Mass Spectrometry. Anal. Chem. 2005, 77, 5370-5379.

30. Hogan, C. J.; Carroll, J. A.; Rohrs, H. W.; Biswas, P.; Gross, M. L. Charge Carrier Field Emission Determines the Number of Charges on Native State Proteins in Electrospray Ionization. J. Am. Chem. Soc. 2008, 130 6929-6927.

31. Konermann, L. A Minimalist Model for Exploring Conformational Effects on the Electrospray Charge State Distribution of Proteins. J. Phys. Chem. B 2007, 111, 6534-6543.

32. Rayleigh, L. On the Equilibrium of Liquid Conducting Masses Charged with Electricity. Phil. Mag. 1882, 14, 184-186.

33. Fenn, J. B. Electrospray Wings for Molecular Elephants (Nobel Lecture) Angew. Chem. Int. Ed. 2003, 42, 3871-3894.

34. Consta, S. Fragmentation Reactions of Charged Aqueous Clusters. J. Mol. Struct. (Theochem.) 2002, 591, 131-140.

35. Last, I.; Levy, Y.; Jortner, J. Beyond the Rayleigh Instability Limit for Multicharged Finite Systems: From fission to Coulomb Explosion. Proc. Natl. Acad. Sci. U.S.A. 2002, 99, 9107-9112.

36. Duft, D.; Lebius, H.; Huber, B. A.; Guet, C.; Leisner, T. Shape Oscillations and Stability of Charged Microdroplets. Phys. Rev. Lett. 2002, 89, 0845031-0845034

37. Li, K.-Y.; Tu, H.; Ray, A. K. Charge Limits on Droplets during Evaporation. Langmuir 2005, 21, 3786-3794.

38. Ntamack, G. E.; Huber, B. A.; Chandezon, F.; Njock, M. G. K.; Guet, C. Simulation of Mass Spectra Produced in Collisions of Highly Charged Ions with Sodium Clusters. J. Phys. B At. Mol. Opt. Phys. 2002, 35, $2729-2740$.

39. Lyalin, A. G.; Obolensky, O. I.; Greiner, W. Stability of Charged Atomic Clusters. Rom. Rep. Phys. 2007, 59, 499-513.

40. Möller, P.; Madland, D. G.; Sierk, A. J.; Iwamoto, A. Nuclear Fission Modes and Fragment Mass Asymmetries in a Five-Dimensional Deformation Space. Nature 2001, 401, 785-790.

41. Hanggi, P.; Talkner, P.; Borkovec, M. Reaction-Rate Theory: Fifty Years after Kramers. Rev. Mod. Phys. 1990, 62, 251-342.

42. Echt, O.; Märk, T. D. Multiply Charged Clusters. In Clusters of Atoms and Molecules II, Haberland, H., Ed.; Springer: Berlin Heidelberg/New York, 1994; pp. 183-220.

43. Fung, A.; Li, P.; Godoy-Ruiz, R.; Sanchez-Ruiz, J. M.; Munoz, V. Expanding the Realm of Ultrafast Protein Folding: ppW, a Midsize Natural Single-Domain $\alpha+\beta$ Topology that Folds Downhill. J. Am Chem. Soc. 2008, 130, 7489-7495.

44. Bohr, N.; Wheeler, J. A. The Mechanism of Nuclear Fission. Phys. Rev. 1939, 56, 426-450.

45. Ryce, S. A.; Wyman, R. R. Two Sphere Model for the Asymmetric Division of Electrically Charged Liquid Drops. Can. J. Phys. 1970, 48, 2571-2576.

46. Csiszar, S.; Thachuk, M. Using Ellipsoids to Model Charge Distributions in Gas-Phase Protein Complex Ion Dissociation. Can. J. Chem. 2004, 82 , 1736-1744.

47. Sinelnikov, I.; Kitova, E. N.; Klassen, J. S. Influence of Coulombic Repulsion on the Dissociation Pathways and Energetics of Multiproton Complexes in the Gas Phase. J. Am. Soc. Mass Spectrom. 2007, 18, 617-631.

48. Schwartz, B. L.; Bruce, J. E.; Anderson, G. A.; Hofstadler, S. A.; Rockwood, A. L.; Smith, R. D.; Chilkoti, A.; Stayton, P. S. Dissociation of Tetrameric Ions of Noncovalent Streptavidin Complexes Formed by Electrospray Ionization. J. Am. Soc. Mass Spectrom. 1995, 6, 459-465.

49. Kebarle, P. A Brief Overview of the Present Status of the Mechanism Involved in Electrospray Mass Spectrometry. J. Mass Spectrom. 2000, 35, $804-817$.

50. Terrell, L. An introduction to Statistical Thermodynamics; Dover: Mineola, NY, 1986.

51. de la Mora, J. F. On the Outcome of the Coulombic Fission of a Charged Isolated Drop. J. Colloid Interface Sci. 1996, 178, 209-218.

52. Taflin, D. C.; Ward, T. L.; Davis, E. J. Electrified Droplet Fission and the Rayleigh Limit. Langmuir 1989, 5, 376-384.

53. Fong, C. S.; Black, N. D.; Kiefer, P. A.; Shaw, R. A. An Experiment on the Rayleigh Instability of Charged Liquid Drops. Am. J. Phys. 2007, 75, 499-503. 